\title{
Pulse Shape Analysis and Discrimination for Silicon-Photomultipliers in Helium-4 Gas Scintillation Neutron Detector
}

\author{
Cathleen Barker, Ting Zhu, Lucas Rolison, Scott Kiff, Kelly Jordan, Andreas Enqvist
}

\begin{abstract}
Using natural helium (helium-4), the Arktis 180-bar pressurized gas scintillator is capable of detecting and distinguishing fast neutrons and gammas. The detector has a unique design of three optically separated segments in which 12 silicon-photomultiplier (SiPM) pairs are positioned equilaterally across the detector to allow for them to be fully immersed in the helium-4 gas volume; consequently, no additional optical interfaces are necessary. The SiPM signals were amplified, shaped, and readout by an analog board; a $250 \mathrm{MHz}$, 14-bit digitizer was used to examine the output pulses from each SiPMpair channel. The SiPM over-voltage had to be adjusted in order to reduce pulse clipping and negative overshoot, which was observed for events with high scintillation production. Pulse shaped discrimination (PSD) was conducted by evaluating three different parameters: time over threshold (TOT), pulse amplitude, and pulse integral. In order to differentiate high and low energy events, a 30ns gate window was implemented to group pulses from two SiPM channels or more for the calculation of TOT. It was demonstrated that pulses from a single SiPM channel within the $30 \mathrm{~ns}$ window corresponded to low-energy gamma events while groups of pulses from two-channels or more were most likely neutron events. Due to gamma pulses having lower pulse amplitude, the percentage of measured gamma also depends on the threshold value in TOT calculations. Similarly, the threshold values were varied for the optimal PSD methods of using pulse amplitude and pulse area parameters. Helium-4 detectors equipped with SiPMs are excellent for in-the-field radiation measurement of nuclear spent fuel casks. With optimized PSD methods, the goal of developing a fuel cask content monitoring and inspection system based on these helium-4 detectors will be achieved.
\end{abstract}

Index Terms-SiPM, helium-4 detector, pulse shaped discrimination, digitizer

This paper was submitted to conference proceedings on 26 May 2017. This material is in part based upon work supported by the Department of Energy Nuclear Engineering University Program (NEUP) under Award Number DE-NE0008459. Additionally, the United States Army paid for C. Barker's master's degree in Nuclear Engineering from the University of Florida.

C. Barker was with the University of Florida, Gainesville, FL 32606 USA and is now with the United States Military Academy, West Point, NY, 10996 USA (e-mail: cathleen.b.barker.mil@mail.mil).

T. Zhu (email: ting.zhu@ufl.edu), L. Rolison (email: kassodus@ufl.edu), K. Jordan (email: kjordan@mse.ufl.edu), and A. Enqvist (email: enqvist@mse.ufl.edu) are with the University of Florida, Gainesville, FL 32606 USA. S. Kiff is with Sandia National Laboratory (email: skiff@sandia.gov).

\section{INTRODUCTION}

$T$ HE Nuclear Engineering Department at the University of Florida has already done a considerable amount of research into neutron spectrometry using helium-4 gas scintillation detectors with PMT readout, developed by Arktis, in order to support research goals from the Department of Energy [1]. In contrast, this research delves into Arktis' new generation of siliconphotomultiplier (SiPM)-based helium-4 detectors in order to analyze their discrimination capabilities and neutron spectrometry for future use in fuel cask content monitoring and inspection systems. Recent studies have noted SiPMs' many useful advantages such as good signal gain, low operation voltage, compactness, and low sensitivity to vibration and magnetic fields [2]. This makes them an ideal detector for the rugged requirements of field radiation measurements.

Using natural helium $\left({ }^{4} \mathrm{He}\right)$, the Arktis 180-bar pressurized gas scintillator is capable of detecting and distinguishing fast neutrons and gammas. An extended version of the detector has an additional ${ }^{6} \mathrm{Li}$-based lining, which allows for detecting thermal neutrons. The most unique feature of the Arktis helium-4 SiPM, shown in Fig. 1, is its $12 \mathrm{SiPM}$ pairs positioned equilaterally across the detector. These pairs are divided into three segments, labeled as segments 1, 2, and 3; all three segments are fully immersed in the helium- 4 gas volume. Therefore, no additional optical interfaces are needed. A readout board, shown with the cover removed in Fig. 2., provides signals from individual SiPM-pair channels which are amplified and shaped by internal electronics [3]. 


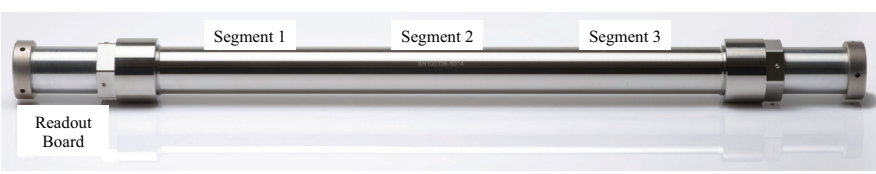

Fig. 1. Example of Arktis helium-4 SiPM detector with readout board (Arktis Radiation Detectors Ltd., 2015).

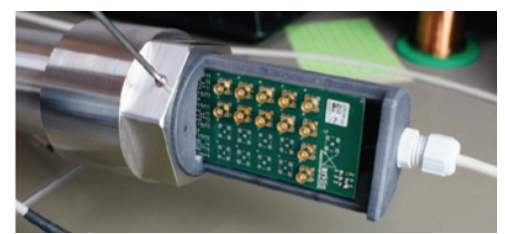

Fig. 2. Readout (analog) board with the cover removed.

\section{MeAsurement SET-UP}

A MATLAB script was created to analyze the data measured by the Arktis helium-4 SiPM detector for pulse shaped discrimination. The purpose of PSD is to examine the effectiveness of the Arktis helium-4 SiPM detector's ability to distinguish between gamma and neutron events. Three separate criteria were evaluated in order to determine which method had the best separation spread: time-over-threshold, peak amplitude, and pulse integral.

The Struck SIS3316-DT, $250 \mathrm{MHz}$, 14-bit digitizer [4], as shown in Fig. 3., consisting of four banks with a total of 16 channels was utilized in all experiments. Each detector segment (i.e. 4 SiPMpair channels) can be connected to fill one bank of the digitizer.

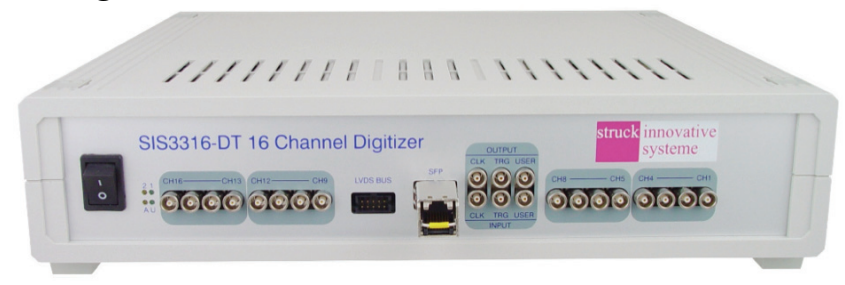

Fig. 3. Struck SIS3316-DT 16-channel digitizer.

Measurements were taken at Sandia National Laboratory using Co-60, Cf-252, AmBe and AmLi sources. Cf-252 was placed inside HDPE spheres of increasing radii in order to assess the thermal neutron detector capability of the extended-range detector which contains the ${ }^{6} \mathrm{Li}$-based lining.. AmLi neutron energy spectrum peaks at lower neutron energy levels than Cf-252. In contrast, the AmBe neutron energy spectrum has much higher neutron energies than Cf-252 [5]. Therefore, using three different neutron sources would provide a wider-range of neutron data for PSD analysis.
Finally Arktis' standard applied voltage for SiPM detectors is $30.5 \mathrm{~V}$; however, some experiments conducted at the University of Florida examined the effects of lowering SiPM gain for discriminating low-energy gamma events by applying a lower voltage of $29.0 \mathrm{~V}$.

\section{A. Time-over-Threshold PSD}

According to the Arktis manual [6], the SiPM controller uses the time-over-threshold (TOT) method to distinguish gamma, thermal, and fast neutron events. The manual specifies that a $30 \mathrm{~ns}$ coincidence window is utilized to group pulses from two or more SiPM channels together. During postprocessing, a threshold value is then applied to the grouped pulses. As long as one pulse from the grouping is "active" (above the threshold), the length of time from the second-to-last instance to the second instance is measured. This is demonstrated in Fig. 4.

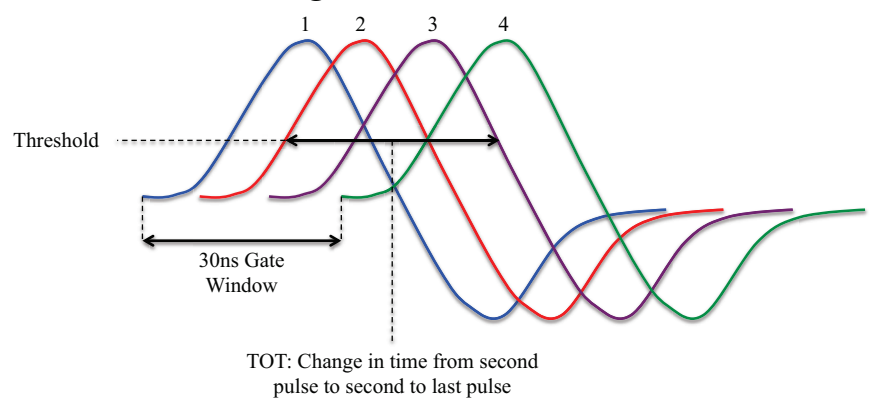

Fig. 4. Time-Over-Threshold (TOT) is defined as the time duration for SiPM pulse signals sustaining a level over a given threshold. A coincident window (default 30ns) can be set to group pulses for a single incident particle event.

In this work, the Time-Over-Threshold parameter is defined as the time duration of consecutive SiPM pulses which sustain a level higher than a given signal threshold. A coincident window of $30 \mathrm{~ns}$ (by Arktis' default) can be imposed and for groups of two or more pulses, they are denoted as the "groups of multiples" and the single pulses that do not fit into the 30 ns gate window are "groups of singles". In our work, at least three thresholds were considered: a low, medium, and a high threshold for each set of data.

\section{B. Peak Amplitude and Pulse Integral PSD}

This research delved into two additional variables: peak amplitude and area in addition to the TOT method by Arktis..

Peak amplitude is the peak amplitude (relative to 
the given threshold level) achieved for an individual pulse. Results in this paper are reported in digitizer units (ie. a dynamic range of $2 \mathrm{~V}$ over $2^{14}$ bit for the Struck digitizer) unless specified otherwise. The area parameter is defined as the integrated area of the pulse in units of digitizer unit times nanoseconds.

Peak amplitude and area of the pulses were evaluated for with and without a 30 ns gate window applied. Like TOT, when a 30 ns gate window was applied, this research evaluated the peak amplitude and the area for both the groups of "multiples" and the groups of "singles."

A variation on the peak amplitude was evaluated when the 30 ns gate was applied. For groups of two or more pulses, the greatest peak amplitude was selected. Additionally, for groups of two or more pulses, the sum of the peak amplitudes for all the pulses in that grouping was evaluated. Furthermore, the area was summed for all pulses grouped together due to the 30 ns gate window.

\section{RESULTS}

The focus of the PSD was to evaluate the effectiveness of TOT, Arktis' specified PSD method, and look at other pulse discriminating qualities to distinguish gammas and neutrons.

\section{A. Effect of Threshold Value for TOT}

Fig. 5 shows a 100-pulse example of Cf-252 measured at the Sandia National Laboratory; various threshold lines have been added to the figure.

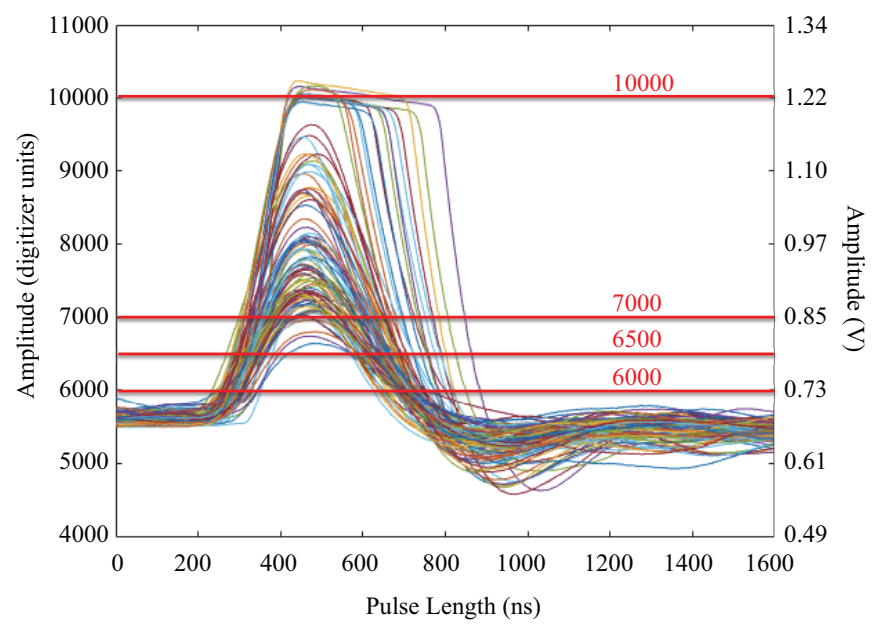

Fig. 5. Depiction of $100 \mathrm{Cf}-252$ pulses collected. Note the saturation of SiPM pulse above 10000 digitizer unit.
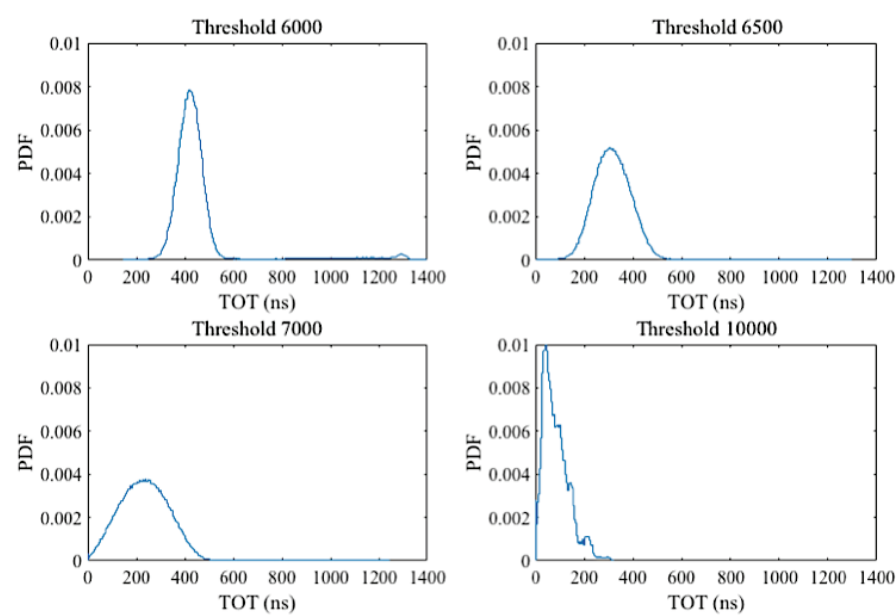

Fig. 6. Quad chart of Cf-252 using data collected from Sandia National Laboratory showing the effects on TOT for different threshold values.

If the threshold is too low, the discrimination ability of TOT for gamma and neutron events is limited. Using an extremely high-value above baseline thresholds, such as at 10000 digitizer units, does two critical things: it removes gamma events almost completely from the data and the TOT distribution will artificially decrease which makes any remaining gamma events difficult to process. This is shown in Fig. 6.

\section{B. TOT with and without coincident window}

Initially, TOT for all individual pulses without using a 30 ns coincident gate window was evaluated. Fig. 7 shows Cf-252, AmLi, AmBe, Co60 , and a background measurement from Sandia National Laboratory using threshold 6500. The majority of Co-60 has a lower TOT distribution than the neutron sources. This indicates that lower TOTs, if the threshold setting is optimized, are more likely to be gamma events than higher TOTs.

However, there is a secondary Co-60 TOT peak that corresponds to the background TOT distribution. This indicates the helium-4 SiPM detector is sensitive to higher energy events such as cosmic radiation. The SiPM's ability to detect background neutrons and higher energy events was further examined at the University of Florida. 


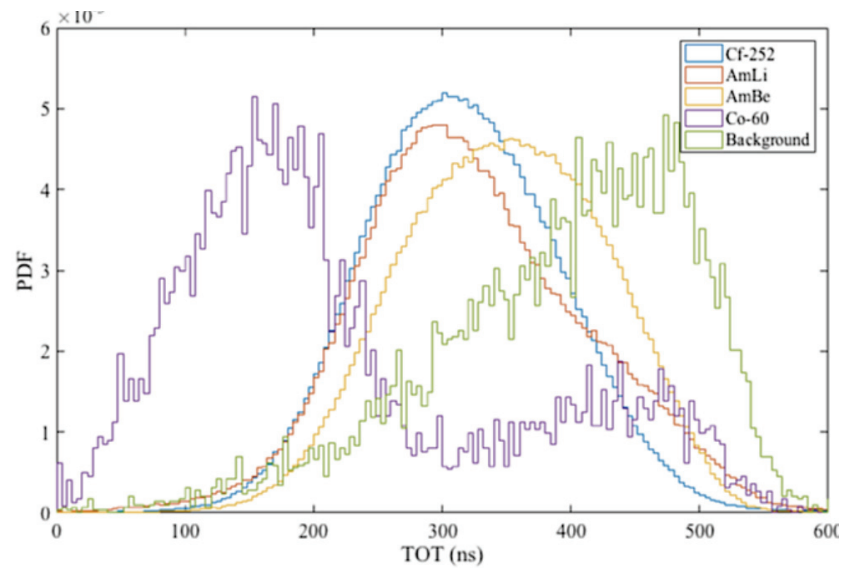

Fig. 7. Quad chart of Cf- 252 using data collected from Sandia National Laboratory showing the effects on TOT for different threshold values.

Although there is promising distinction between TOT for individual pulses collected from gamma and neutron sources, Arktis specifies that they use a 30 ns gate window for their TOT evaluation. As long as two or more events are above the threshold value within a $30 \mathrm{~ns}$ window, then TOT is measured. When this $30 \mathrm{~ns}$ gate window was applied, pulses that were within the $30 \mathrm{~ns}$ gate window were grouped together and are referred to as groups of "multiples." Pulses that were not within 30 ns of another pulse are referred to as groups of "singles." Fig. 8 displays the TOT for groups of "singles" while Fig. 9 displays the TOT for groups of "multiples."

Overall, the neutron sources had higher percentages of groups of "multiples," ranging between $37-49 \%$ compared to the Co-60 source. In contrast, Co-60, a gamma source, only $12 \%$ are groups of "multiples." $88 \%$ of Co-60 was found to be in groups of "singles." This shows that the $30 \mathrm{~ns}$ gate window discriminates between neutron and gamma sources. Furthermore, 51\% of the background pulses are in groups of "multiples" which indicate they are higher energy events. This supports the theory that the majority of the background data were from cosmic rays.

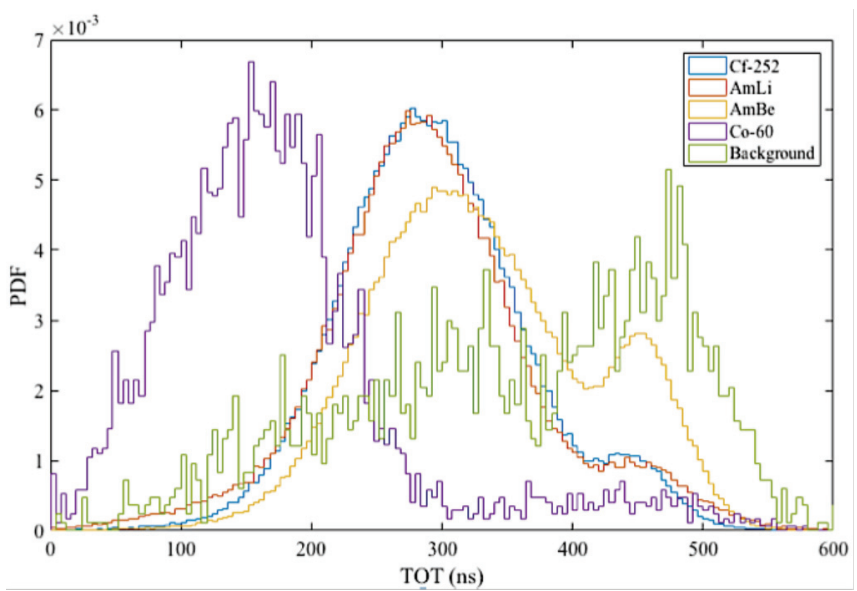

Fig. 8. Quad chart of Cf-252 using data collected from Sandia National Laboratory showing the effects on TOT for different threshold values.

Fig. 8 displays the groups of "singles" which shows a difference in TOT distribution for gamma and neutron sources. There is a secondary TOT distribution peak in the neutron data at higher TOT values. This peak corresponds to the background TOT distribution.

Fig. 9 displays the TOT distribution for groups of "multiples." Here, there are no lower-value TOTs. The Co-60 TOT distribution matches the background TOT distribution for groups of "multiples." Additionally, the rate for groups of "multiples" for Co-60 and background are the within 0.01 groups $/ \mathrm{sec}$ of each other. This indicates that all of the Co- 60 measured within the $30 \mathrm{~ns}$ coincident window are actually background. Therefore, the events that make the 30 ns gate window are either neutrons or high-energy background events.

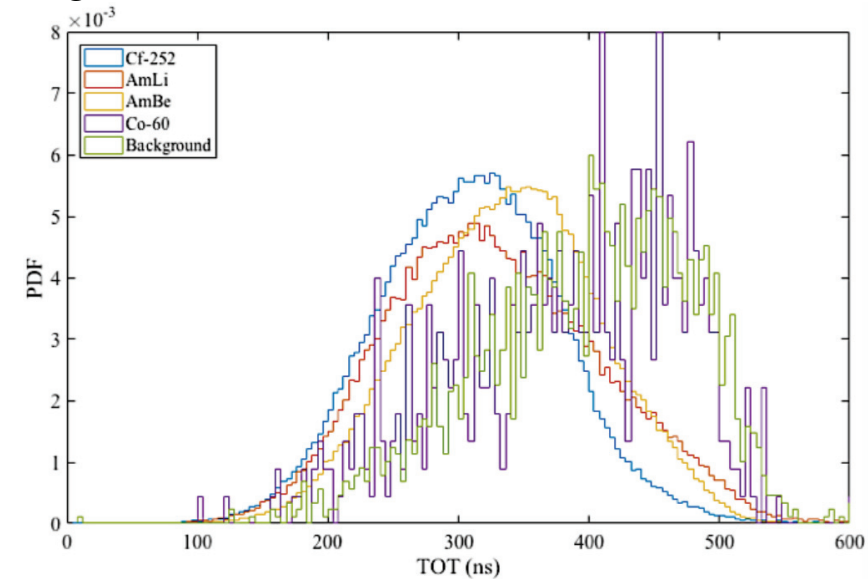

Fig. 9. Quad chart of Cf-252 using data collected from Sandia National Laboratory showing the effects on TOT for different threshold values.

\section{PSD for each segment}

Analysis of the scintillation pulses in each 
segment for TOT, peak amplitude, and integral showed minor discrepancies among the three detector segments. For example, a Cf-252 source was positioned $0.4 \mathrm{~m}$ from the center of detector's segment 2. The distributions of the TOT values for SiPM pulses for each segment are shown in Fig. 10. It was expected and confirmed that segment 2 had a greater amount of measured events. However, Fig. 10 shows that segment 1 measured significantly more events than segment 3 and has a lower TOT peak value.

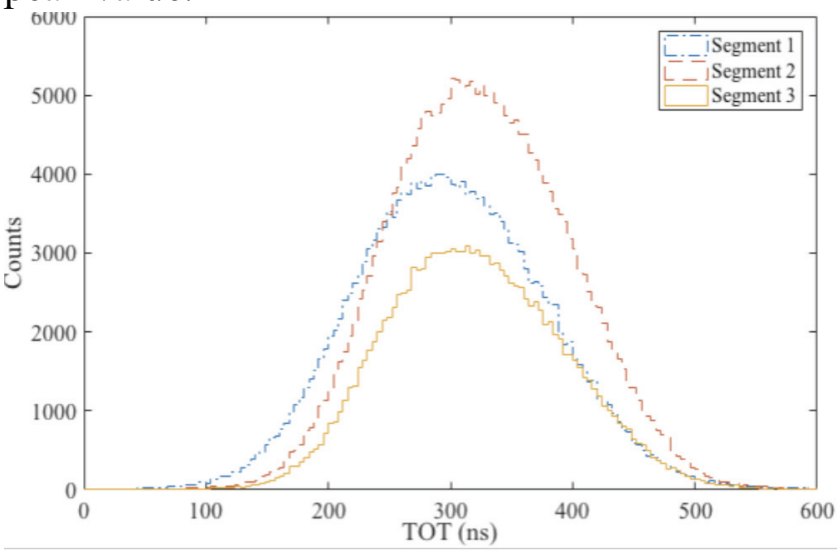

Fig. 10. TOT for individual pulses for all three segments using data collected from Sandia National Laboratory

This discrepancy between segments 1 and 3 is also observed in Figs. 11 and 12, which show the distributions of the values of SiPM pulse peak amplitude and pulse integrated area in each segment.

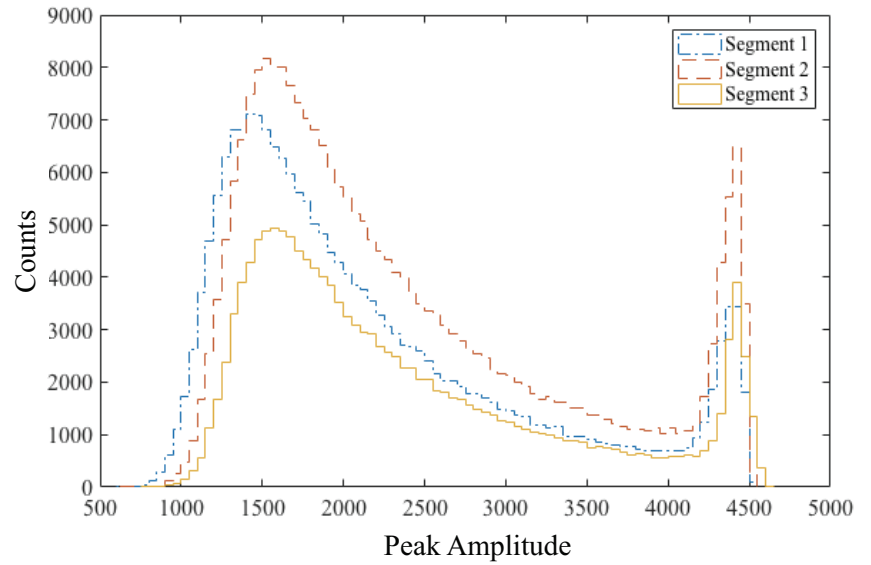

Fig. 11. Distributions of SiPM pulse peak amplitude values for all three segments using a Cf-252 source aligned with segment 2 center and with equal distant to segments 1 and 3 .

Overall, Figs. 10-12 indicate that either there may be some inconsistency of scintillation characteristics among the three segments of the detector, which are supposedly optically independent and identical.

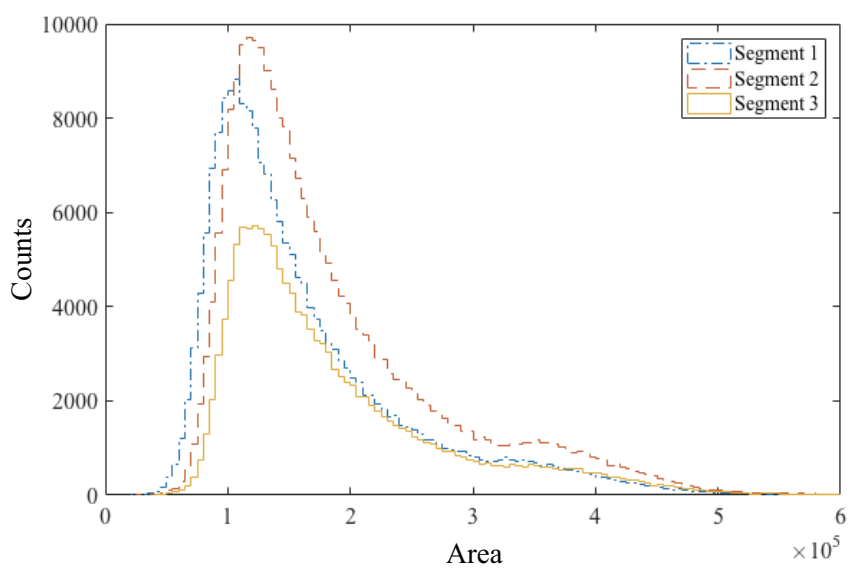

Fig. 12. Distribution of SiPM pulse integrated area values for all three segments using a Cf-252 source aligned with segment 2 center and with equal distant to segments 1 and 3 .

\section{TOT comparison between $30.5 \mathrm{~V}$ and $29.0 \mathrm{~V}$}

Additional research examined the effect of decreasing the applied voltages on the effectiveness of PSD. The nominal supply voltage for the SiPM detector electronics is $30.5 \mathrm{~V}$. As shown in Fig. 13, the distributions of TOT for the Cf-252, Co-60 and a background measurement are distinct and demonstrate good PSD capability of the detector. Additionally, there is a small secondary peak in the Co-60 measurement that has a TOT peak around 525 ns. When evaluating the background, it is apparent that the $525 \mathrm{~ns}$-peak corresponds to background. However, at a lower supply voltage of $29.0 \mathrm{~V}$, the count rates for Co-60 and the background are nearly the same. Comparing Fig. 13 and Fig. 14, there is a shift of the distributions to lower TOT values in general since the same threshold value was applied, but the SiPM pulses' amplitudes were decreased due to the lower voltage. In Fig. 13, the matching TOT distribution peaks of Co-60 and background implies that the actual gamma events from Co-60 were not measured by the detector; instead the same background contribution was measured. This is a positive finding because helium- 4 based SiPM detectors are being considered for spent nuclear fuel detection; lowering the applied voltage can theoretically remove the SiPM detector's capability to measure gamma events. 


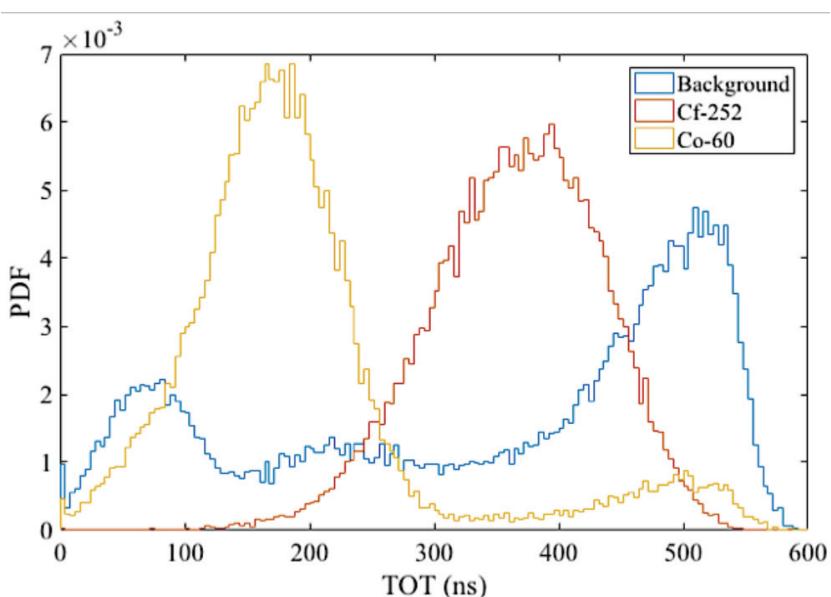

Fig. 13. TOT for individual pulses at $30.5 \mathrm{~V}$ using data collected from University of Florida. Note the measurement durations were different for the three cases here.

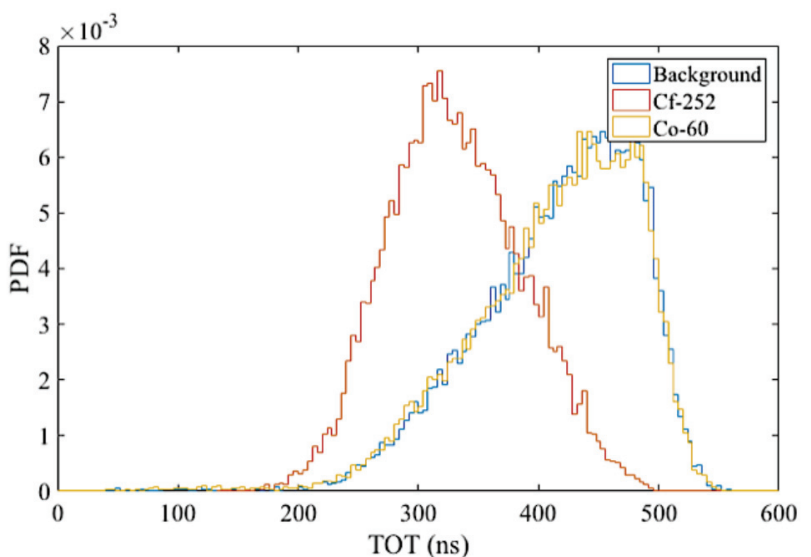

Fig. 14. TOT for individual pulses at $29.0 \mathrm{~V}$ using data collected from University of Florida.

The effect of lowering supply voltage on the distribution of pulse peak values is shown in Fig. 15. At $30.5 \mathrm{~V}$, there is a large background peak at the clipped pulse region (around 4500 digitizer amplitude units) due to SiPM pulse saturation, as noted in Fig. 5. There are similar peaks for both Cf252 and Co-60. At $29.0 \mathrm{~V}$, the Cf- 252 peak in that region is almost completely removed. The Co- 60 peak matches the background peak in that region. This shows that lowering the applied voltage will remove clipped pulses but also suppress low-energy gamma event measurement.
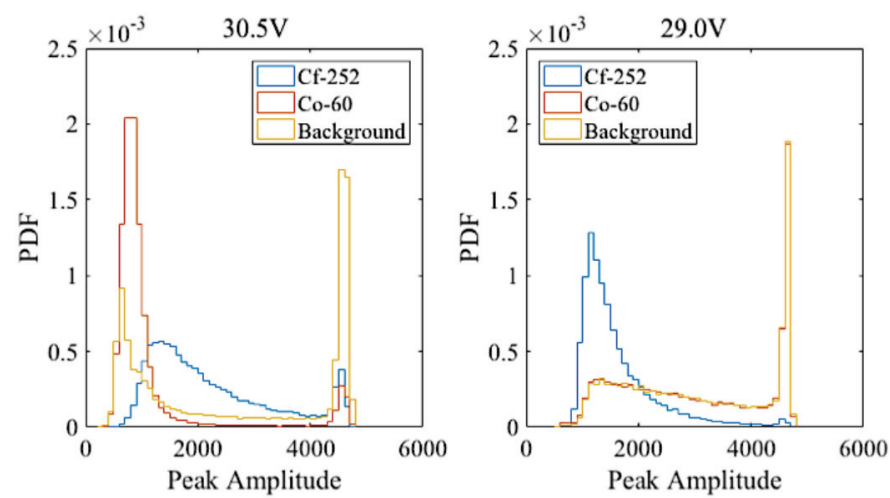

Fig. 15. Peak amplitude for individual pulses at $30.5 \mathrm{~V}$ and $29.0 \mathrm{~V}$ using data collected from University of Florida.

\section{E. TOT comparison with longer coincident window}

Results above were obtained by using individual SiPM pulse data, without taking into account the fact that one event could induce multiple SiPM pulses. As mentioned, a coincidence window can be applied to group consecutive pulses together. Window lengths of $30 \mathrm{~ns}, 60 \mathrm{~ns}, 100 \mathrm{~ns}, 200 \mathrm{~ns}$, and $400 \mathrm{~ns}$ were used and the results are presented in Fig. 16.

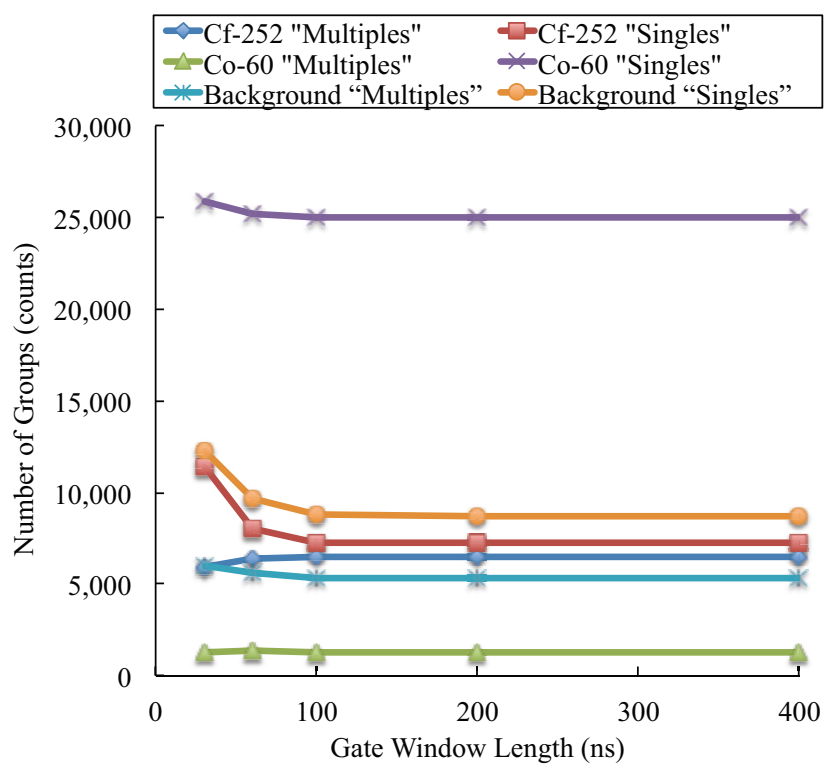

Fig. 16. Number of groups versus coincident window lengths for $\mathrm{Cf}-252$, Co60 , and background.

The groups of "singles' were events where there was only one recorded SiPM pulse in the given coincident window length. The decrease in the number of groups of "singles" in all cases of Cf252, Co-60, and the background as the window length increase simply means that some single events are grouped together; thus, it is likely that the gate window is "over-cautious" in preventing a false positive (calling an event a neutron when it is 
really a gamma). Eventually, however, the trend becomes stable for all three measurements, as the gate window length is increased. This shows that the rate of false positives does not increase for gate window lengths greater than $100 \mathrm{~ns}$.

Fig. 17 further evaluates this conclusion by comparing the TOT for $30 \mathrm{~ns}$ and $100 \mathrm{~ns}$. The TOT values for groups of "singles" for both $\mathrm{Cf}-252$ and Co-60 stay the same. The TOT for background "singles" looks considerably different at $100 \mathrm{~ns}$ than at $30 \mathrm{~ns}$. The peak around $500 \mathrm{~ns}$ for background "singles" for the $30 \mathrm{~ns}$ data is completely missing from the $100 \mathrm{~ns}$ data. Larger TOT tends to indicate that the event is a neutron or other high-energy event but not every high TOT is a neutron event. Therefore, the rate of false positives has likely increased as a result of increasing the gate window length.
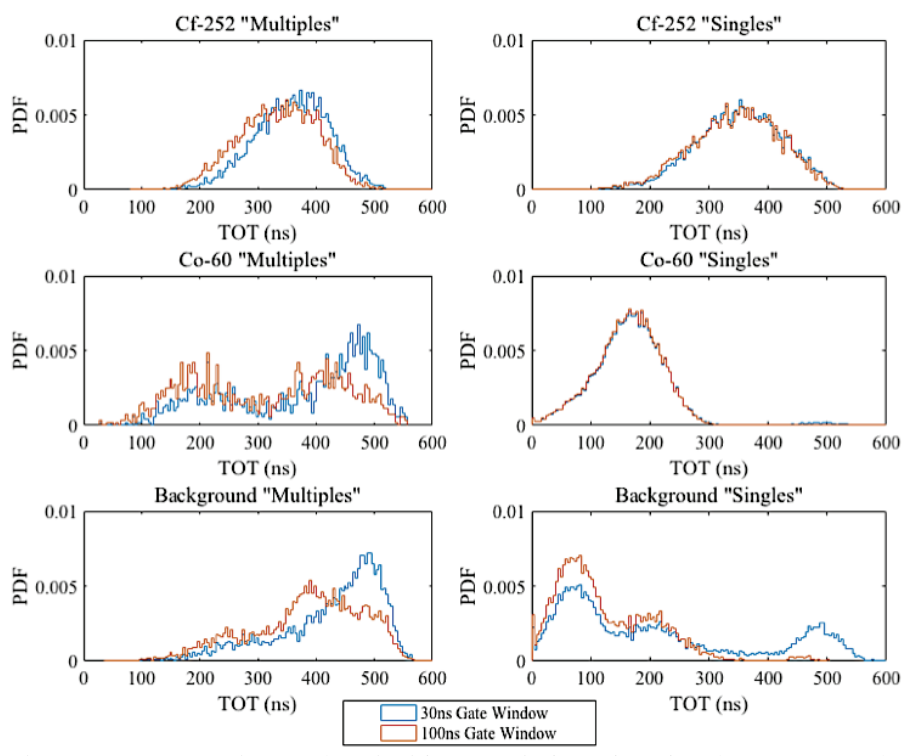

Fig. 17. Comparison of coincidence window lengths for groups of "multiples" and "singles".

Another important note is that although groups of "multiples" increased for Cf-252 and Co-60, the distribution has shifted to a lower TOT value. The Co-60 distribution has the largest shift of TOT in the 400-600ns range. Although counterintuitive, this could be attributed to the number of groups of "multiples" increasing but the average number of pulses in each group decreasing. Fewer pulses in a group will, in general, have a shorter TOT. This a positive effect because it helps distinguish Co-60 group of "multiples" TOT from Cf-252 groups of multiples." Consequently, gate window can be increased slightly with some minimal, but positive, effects on TOT comparison.

\section{CONClusion}

Pulse shaped discrimination analysis were conducted on data collected by Arktis helium-4 SiPM detectors. There are clearly many measurement settings that must be adjusted prior to collecting data in order to get the clearest results. Modifications to signal threshold value and supply voltage will impact the ability during postprocessing to distinguish gamma and neutron events.

The PSD analysis showed several things. First, TOT has the clearest distinction between gamma and neutron events when compared with peak amplitude and pulse area analysis. Secondly, decreasing the applied voltage improves the helium4 SiPM detector's ability to suppress gamma events. Furthermore, a $30 \mathrm{~ns}$ coincidence window length provides better discrimination between gamma and neutron events than looking at each pulse individually. Finally, the gate window does have some room to be increased from $30 \mathrm{~ns}$ to $100 \mathrm{~ns}$, which can improve gamma and neutron discrimination, except in very high-rate environments where pulse-pileup could become non-negligible with increased gate window.

However, with these positive trends, more research is needed to fully characterize the capabilities of the Arktis helium-4 SiPM detectors. PSD-focused measurements were only conducted using the "non-extended range" version of the detectors. The "extended range" version theoretically should show distinction in the groups of "multiples" between thermal and fast neutrons. Additionally, longer measurements would increase the accuracy of the results and more extensive research needs to be conducted to refine the limits of the coincidence window for TOT. Finally, further research needs to be conducted in order to utilize the PSD method to develop a neutron response matrix. 


\section{REFERENCES}

[1] R. P. Kelley, et al., "Neutron response function characterization of $4 \mathrm{He}$ scintillation detectors," Nuclear Instruments and Methods in Physics Research A, vol. 793, pp. 101-107, 2015.

[2] P. Buzhan, et al., "Silicon photomultiplier and its possible applications," Nuclear instruments and Methods in Physics Research Section A, vol. 504, pp. 48-52, May 2003.

[3] Arktis Radiation Detectors Ltd., "Analog Readout Board AA121 Assembly and Operating Manual," Mar. 12, 2015. [Online]. Available: www.arktis-detectors.com.

[4] "SIS3316, 16 Channel VME Digitizer User Manual," Struck Innovative Systeme, Hamburg, Germany, January 2015.

[5] D. Reilly, N. Ensslin, \& H. Smith, Jr., "Passive Nondestructive Assay of Nuclear Materials," Los Alamos National Laboratory, Mar. 1991. [Online]

http://www.lanl.gov/orgs/n/n1/FMTTD/neut_mc /pdfs/LA_UR_90_0732.pdf

[6] Arktis Radiation Detectors Ltd., "Arktis S-670 Detector Series Operating Manual," Mar. 12, 2015. [Online]. Available: www.arktisdetectors.com. reaction. Journal of Radioanalytical and Nuclear Chemistry , 256 (1), 163-166. 